\title{
Atrophy of the corpus callosum associated with a decrease in cortical benzodiazepine receptor in large cerebral arterial occlusive diseases
}

H Yamauchi, H Fukuyama, Y Dong, H Nabatame, Y Nagahama, S Nishizawa, J Konishi, H Shio

\begin{abstract}
Objectives-It remains controversial whether selective neuronal ischaemic change develops in patients with occlusion of the large cerebral arteries. Previous studies have shown atrophy of the corpus callosum with reduced cortical oxygen metabolism in large cerebral arterial occlusive diseases, which might be indirect evidence of loss of the neurons in cortical layer 3. Recent studies of patients with ischaemic cerebrovascular diseases have demonstrated reduced central benzodiazepine receptor (BZR) binding in the normal appearing cortical areas, which might be more direct evidence of changes of the neurons. Although pathophysiology of the decreased BZR is unclear, a decrease in the cortical BZR binding with neuronal loss would cause atrophy of the corpus callosum. The purpose of this study was to determine whether atrophy of the corpus callosum is associated with a decrease in cortical BZR binding in large cerebral arterial occlusive diseases.

Methods-Seven patients with occlusive diseases of the middle cerebral or internal carotid artery and only minor subcortical infarctions were studied. Single photon emission tomographic images of ${ }^{123} \mathrm{I}$ labelled iomazenil (IMZ) obtained $180 \mathrm{~min}-$ utes after injection were analysed for BZR binding. The midsagittal corpus callosum area/skull area ratio (on $\mathrm{T} 1$ weighted magnetic resonance images) was compared with the cerebral IMZ uptakel cerebellar IMZ uptake ratio.
\end{abstract}

Results-Compared with 23 age and sex matched control subjects, the patients had significantly decreased callosal area/skull area ratio. The degree of corpus callosum atrophy was significantly and strongly $(\rho=0.99, p<0.02)$ correlated with that of the decreases in the mean cerebral cortical IMZ uptake ratio.

Conclusion-Corpus callosum atrophy may occur in association with a decrease in cortical BZR binding in large cerebral arterial occlusive diseases. Corpus callosum atrophy with decreased cortical BZR binding might reflect cortical neuronal damage in large cerebral arterial occlusive diseases.

(7 Neurol Neurosurg Psychiatry 2000;68:317-322)

Keywords: benzodiazepine receptors; corpus callosum; cerebrovascular disorders; carotid artery disease
In patients with large cerebral arterial occlusive diseases, low perfusion or microembolism may produce ischaemic changes that are not detectable as infarction on CT or MRI. Neuropathological studies have demonstrated cortical granular atrophy or watershed territory mocroemboli in the cerebral cortex distal to large cerebral arterial occlusion. ${ }^{1}$ In addition, recent observations in both humans and animals also suggest that selective cortical neuronal loss results from occlusion of the large cerebral arteries accompanied by ischaemia of moderate severity. ${ }^{3}$ However, it remains controversial whether selective neuronal ischaemic change develops in patients with occlusion of the large cerebral arteries, because a direct demonstration of the cortical neuronal loss in the living brain is not possible.

To consider the issue of neuronal loss without infarction in large cerebral arterial occlusive diseases, indirect markers for neuronal damage including brain atrophy and hypometabolism have been investigated. Atrophy of the corpus callosum may be an indirect but sensitive indicator of ischaemic cortical neuronal loss. The largest fraction of neurons projecting into the corpus callosum is the large pyramidal cells in layer $3,{ }^{4}$ which is one of the groups of cortical neurons most vulnerable to ischaemia. ${ }^{1}$ Therefore, callosal atrophy may result from cortical ischaemic damages. Reduced cerebral cortical oxygen metabolism without morphological changes, which surgical reperfusion can not improve, has been demonstrated in occlusive diseases of the internal carotid artery (ICA) or middle cerebral artery (MCA) by using PET, suggesting that some irreversible damage may occur. ${ }^{5-8}$ Our previous studies showed that a decrease in bilateral cerebral cortical oxygen metabolism occurs in association with callosal atrophy in patients with ICA occlusive disease, suggesting that ischaemic neuronal loss may contribute to the reduced cortical oxygen metabolism. ${ }^{9-11}$ However, to consider this issue further, a more direct marker for neuronal damage was needed.

Recent development of imaging techniques makes it possible to visualise the distribution of central type benzodiazepine receptors (BZR) in humans by using ${ }^{11} \mathrm{C}$ labelled flumazenil for PET or ${ }^{123}$ I labelled iomazenil (IMZ) for single photon emission computed tomography (SPECT). ${ }^{12}{ }^{13}$ Benzodiazepine receptors are known to be coupled with $\gamma$-amino butyric acid receptors and chloride channel proteins, and 
central BZRs exist in the membrane of neurons. ${ }^{14}$ Thus, imaging of the central BZRs, which are expressed by most cortical neurons, might detect neuronal damage, which could not be evaluated directly by other neuroimaging modalities. ${ }^{215}$ Recent studies have demonstrated reduction in BZR binding in the normal appearing cortical areas on CT and MRI in patients with cerebrovascular diseases, the degree of which was related to the presence of cortical signs, suggesting that selective neuronal change might be caused by ischaemia. ${ }^{16-21}$ However, pathophysiology of the decreased $\mathrm{BZR}$ is unclear, and the relative contribution to the decreases in BZR binding of reduced receptors on remaining neurons and loss of neurons cannot be determined from the imaging of BZR only.

In this study, we further considered the issue of selective cortical neuronal loss by using callosal atrophy and BZR binding as an indirect marker of neuronal loss and a more direct marker of neuronal change, respectively. If a decrease in the cortical BZR binding in the normal appearing cortical areas reflects ischaemic neuronal loss in large cerebral arterial occlusive diseases, callosal atrophy may accompany it. To our knowledge, no study has systematically analysed the relation between BZR binding and morphological measures of brain atrophy, including callosal size. The objective of this study was to investigate whether atrophy of the corpus callosum is associated with a decrease in cortical BZR binding in large cerebral arterial occlusive diseases.

\section{Subjects and methods}

SUBJECTS

We studied seven consecutive patients with large cerebral arterial occlusive diseases using MRI, SPECT, and PET. All subjects were right handed men aged 57 to 72 years (mean (SD) 63 (6) years). Two patients had transient ischaemic attacks and five had minor stroke with mild disability. Brain MRI disclosed only minor subcortical infarctions defined as well demarcated hypointense areas, $<2 \mathrm{~cm}$ in diameter, on T1 weighted MRI in the MCA territory of the hemisphere with the arterial disease. None of the patients had any symptoms suggesting vertebrobasilar ischaemia, and they showed no cerebellar or brainstem abnormalities on MRI. Conventional angiography showed unilateral MCA occlusion in two patients, unilateral ICA stenosis in two patients ( $75 \%$ and $90 \%$ diameter reduction), unilateral ICA occlusion in two patients, and ICA occlusion with contralateral ICA stenosis $(50 \%)$ in one patient. In the patient with bilateral disease, the symptomatic vascular lesion involved ICA occlusion. The vertebrobasilar system was angiographically normal in all patients. No patient had any history of taking drugs that would affect BZR. Two patients had a history of hypertension, and two others had diabetes mellitus. In all patients, the MRI, SPECT, and PET evaluations were performed within 2 months. The interval between the last symptoms and these evaluations ranged from 4 to 24 months (mean (SD) 12 (8) months). All subjects gave informed consent to the conventional angiography, SPECT, and PET studies.

For the MRI measurements, we studied 23 age matched right handed male subjects, aged 52 to 76 years (mean (SD) 65 (6) years), as controls. Seven had a history of hypertension, and five others had diabetes mellitus. Another six age matched normal male subjects (mean (SD) 61 (2) years), who had no history of medical or psychiatric disorder were used for IMZ SPECT study. ${ }^{18}$ All of these subjects showed normal neurological findings and no specific neurological diseases other than tension type headache. None exhibited any abnormal MRI findings, except for a few punctate high intensity areas of various degrees in the subcortical white matter on T2 weighted images without corresponding abnormality on T1 weighted images.

\section{MRI}

Brain MRI was performed with a unit (Signa, General Electric, Milwaukee, WI, USA) that operated at a field strength of 1.5 Tesla, once for each subject. The T1 weighted sagittal and axial images were obtained using a spin echo pulse sequence (repetition time $400 \mathrm{~ms}$; echo time $15 \mathrm{~ms}$ ). Axial T2 weighted images were also obtained with spin echo pulse sequences (repetition time $3000 \mathrm{~ms}$; echo time $100 \mathrm{~ms}$ ). Axial images were obtained parallel to the orbitomeatal line. The slice thickness was 3 $\mathrm{mm}$ for sagittal images and $5 \mathrm{~mm}$ for axial images. Sections were contiguous in the sagittal plane and had an intersection gap of $1.8 \mathrm{~mm}$ in the axial plane.

The extent of atrophy of the corpus callosum on midsagittal images was measured by using a computer assisted image analyser (FDM98-1; Photron, Tokyo, Japan) and a personal computer (PC-9801; Nihon Electric Co, Tokyo, Japan), as previously described..$^{22}$ In brief, each MR scan (one scan for each subject) was recorded with a video camera and digitised by the image analyser with the use of a $256 \times 256$ data matrix and a 64 step grey scale. For the tracing technique, an outline of each structure was done manually with a mouse cursor. The number of pixels with signal intensities that corresponded to the predetermined level that was set for the region of interest (ROI) was then counted. The level was automatically determined as the range from the maximum pixel value to the mean value of the maximum and minimum values, in which the maximum value was the maximum pixel value of the corpus callosum and the minimum value was that of the background (the CSF space). The total area of the corpus callosum was measured. To control for variation in skull size, we measured the areas of the midline internal skull surface by manually tracing the line through the inner table, foramen magnum, clivus, sellar diaphragm, and jugum sphenoidale, and we then calculated the total callosal area/skull area ratio as a percentage.

In the measurement of the extent of enlargement of the lateral ventricles, the T1 weighted axial section through the image that contained 
the largest extent of the bodies of the lateral ventricles was quantitatively analysed in a similar way. The level that was set for the lateral ventricles was determined as the range from the minimum value to the mean value of the maximum and minimum values minus 1 , where the maximum value was the maximum pixel value of the surrounding white matter and the minimum value was the minimum pixel value of the lateral ventricles. We also measured the areas of the axial internal skull surfaces by tracing the inner table and calculated the lateral ventricular area/skull area ratio as a percentage,separately for each hemisphere.

In the measurement of the extent of atrophy of the cerebral cortex, three T1 weighted axial sections parallel to the orbitomeatal line (corresponding to the levels of the basal ganglia, the body of lateral ventricle, and the centrum semiovale, respectively) were analysed. We measured the area of the subarachnoid space. The level that was set for the subarachnoid space was determined as the range from the minimum value to the mean value of the maximum and minimum values minus 1 , where the maximum value was half the value of the maximum pixel value of the hemispheric white matter and the minimum value was the minimum pixel value of the subarachnoid space. The mean hemispheric value of subarachnoid space area/skull area ratio (as a percentage) was calculated separately for each hemisphere.

All measurements were performed by one investigator who was unaware of the clinical status of the patients. Before this study, the observer reliability of our procedure was evaluated for 20 subjects with or without neurological diseases. There was high intraobserver reliability for the measurement of callosal area/ skull area ratio, lateral ventricular area/skull area ratio, and subarachnoid space area/skull area ratio $(r=0.98, \mathrm{p}<0.001 ; r=0.99, \mathrm{p}<0.001$; and $r=0.98, \mathrm{p}<0.001$, respectively).

To grade white matter lesions, axial T2 weighted images were visually evaluated, separately for each hemisphere. Most of these lesions corresponded to the so called unidentified bright objects. The size of each white matter high intensity lesion, including areas of infarction on $\mathrm{T} 1$ weighted images, was graded from 1 to 9 , with higher numbers indicative of larger size. A score of 1 was given to lesions with diameter $<0.5 \mathrm{~cm} ; 2,1 \mathrm{~cm} ; 3,1.5 \mathrm{~cm} ; 4,2$ $\mathrm{cm} ; 5,2.5 \mathrm{~cm} ; 6,3 \mathrm{~cm} ; 7,3.5 \mathrm{~cm} ; 8,4 \mathrm{~cm}$; and 9 to lesions with diameter $>4 \mathrm{~cm}$. Hemispheric white matter lesion scores were calculated by summing the scores for all lesions in each hemisphere.

SPECT AND PET

For SPECT imaging, a triple head rotating gamma camera (GCA9300A, Toshiba) was used. The spatial resolution was $12 \mathrm{~mm}$ full width half maximum (FWHM) in the centre of the field of the view, and the axial resolution was $23.5 \mathrm{~mm}$ FWHM. Each patient received $222 \mathrm{MBq}$ IMZ by a $1.5 \mathrm{ml}$ intravenous bolus injection. Data acquisition was started at 5 minutes and at 165 minutes after injection and was continued for 30 minutes (early and late IMZ images, respectively). The late images are reported to represent a relative map of BZR binding. ${ }^{23}$ The image was reconstructed as $128 \times 128$ matrix with 16 slices, with each voxel being $2.0 \times 2.0 \times 6.8 \mathrm{~mm}$ in actual size. Another six normal age matched control subjects were examined under a similar protocol.

PET was performed on all patients with the use of a commercially available system (PCT3600W, Hitachi Medical Co, Tokyo, Japan). ${ }^{24}$ This system simultaneously acquires 15 slices with a centre to centre distance of $7 \mathrm{~mm}$. All scans were obtained at a resolution of a $7.5 \mathrm{~mm}$ FWHM in the transaxial direction and $6.5 \mathrm{~mm}$ in the axial direction in the wobbling mode. Patients were positioned with the orbitomeatal line parallel to the detector rings. Before PET was performed, ${ }^{68}$ germanium $/{ }^{68}$ gallium transmission scanning was performed for 20 minutes for attenuation correction. For the ${ }^{15} \mathrm{O}$ gas study, $\mathrm{C}^{15} \mathrm{O}_{2}$ and ${ }^{15} \mathrm{O}_{2}$ were inhaled continuously at $300 \mathrm{MBq}$ and $500 \mathrm{MBq}$ per minute, respectively. The scan time was 5 minutes, and arterial blood was sampled three times during each scan. We calculated cerebral blood flow $(\mathrm{CBF})$, cerebral metabolic rate of oxygen $\left(\mathrm{CMRO}_{2}\right)$, and oxygen extraction fraction (OEF) based on the steady state method. ${ }^{25}$ Inhalation of $1.20 \mathrm{GBq} \mathrm{C} \mathrm{C}^{15} \mathrm{O}$ was used to measure $\mathrm{CBV}$, and $\mathrm{CMRO}_{2}$ and $\mathrm{OEF}$ were corrected with respect to the CBV. ${ }^{26}$ Functional images were reconstructed as $128 \times 128$ pixels in 15 slices, with each voxel representing $2.0 \times 2.0 \times 7.0 \mathrm{~mm}$ in actual size.

Data from SPECT and PET were reconstructed into three dimensional images which were parallel to the orbitomeatal line, and each image consisted of 64 planes with $2 \mathrm{~mm}$ cubic voxels. IMZ SPECT images were coregistered to the CBF image using an SPM95 realignment subroutine. ${ }^{27}$ To compare IMZ SPECT and PET images quantitatively, three sets of SPECT and PET images parallel to the orbitomeatal line (corresponding to the levels of the basal ganglia, the body of lateral ventricle, and the centrum semiovale, respectively) were analysed. Regions of interest (ROIs) were placed on the CBF images, and each image was examined by placing a total of 15 to 17 circular ROIs $12 \mathrm{~mm}$ in diameter compactly over the grey matter of the cortex. ${ }^{8}$ According to the atlas written by Kretschmann and Weinrich, ${ }^{28}$ the ROIs in all three images were included in the distribution of the anterior cerebral artery, MCA, and posterior cerebral artery, as well as the watershed areas between the anterior cerebral artery and MCA (anterior watershed) and between the MCA and posterior cerebral artery (posterior watershed). The mean hemispheric and cerebral values were calculated as the average of the MCA, anterior watershed, and posterior watershed ROI, and each was weighted by region size. The same ROIs were transferred to the IMZ SPECT and $\mathrm{CMRO}_{2}$ images respectively. The ROIs were also drawn on the bilateral cerebellar cortices. ${ }^{29}$ In the calculation of regional relative distribution of central BZR, the cerebellum was used as a reference area, and the relative IMZ uptake 
Table 1 Relative mean cerebral and hemispheric IMZ uptake ratios (\%) and the total corpus callosum area to the skull area ratio (\%) in patients and control subjects

\begin{tabular}{lllll}
\hline & \multicolumn{2}{l}{ Relative IMZ } & & \\
\cline { 2 - 3 } & Mean & Ipsilateral & Contralateral & Corpus callosum Total \\
\hline Normal controls & $119(12)$ & & & $4.17(0.42)$ \\
Patients & $109(4) \dagger$ & $106(6) \ddagger$ & $111(4)$ & $3.31(0.95)^{\star}$ \\
$\%$ Of control value & 91.6 & 89.1 & 93.3 & 79.4 \\
\hline
\end{tabular}

Ipsilateral=Hemisphere ipsilateral to symptomatic arterial diseases; contralateral=the hemisphere contralateral to symptomatic arterial diseases.

Values are mean (SD).

${ }^{\star} \mathrm{p}<0.05 ; \mathrm{p}=0.06 v$ controls (Mann-Whitney $U$ test)

$\ddagger \mathrm{p}<0.05$, versus controls (Kruskal-Wallis test with post hoc Mann-Whitney $U$ test).

(the ratio of the mean hemispheric or cerebral IMZ uptake to the mean IMZ uptake in the bilateral cerebellar cortices) was subjected to analysis.

\section{STATISTICAL ANALYSIS}

Differences between the patients and controls were assessed using a Mann-Whitney $U$ test or a Kruskal-Wallis test with post hoc MannWhitney $U$ test as appropriate. Spearman's rank correlation was used for correlation between the two variables. Statistical significance was accepted at $\mathrm{p}<0.05$. Multiple linear and stepwise regression analyses were used to analyse the relations among the callosal area/skull area ratio, the subarachnoid space area/skull area ratio, the ventricular area/skull area ratio, white matter lesion scores, the relative cerebral cortical IMZ uptake, the values of cortical CMRO2, the patient age, and the time between the last symptoms and the MRI evaluation.

\section{Results}

The total callosal/skull area ratio in patients was significantly decreased compared with that in the controls. The relative cerebral cortical IMZ uptake in the hemisphere ipsilateral to symptomatic arterial diseases was significantly decreased compared with that in the controls. When expressed as a percentage of the control value, the severity of callosal atrophy was greater than that of the decreases of IMZ uptake (table, fig 1).

In the patients, the total callosal/skull area ratio showed a very strong correlation with the relative mean cerebral cortical IMZ uptake $(\rho=0.99, p<0.02$, fig 2$)$. The total callosal/skull area ratio was not significantly correlated with the total white matter lesion scores $(\rho=-0.67$, $\mathrm{p}=0.10$ ). When controlling for the total white matter lesion scores, the correlation between the total callosal/skull area ratio and the relative mean cerebral cortical IMZ uptake was still highly significant (partial correlation coefficient $=0.96, \mathrm{p}<0.005)$.

The relative mean cerebral cortical IMZ uptake was not significantly correlated with the mean cerebral value of subarachnoid space area/skull area ratio $(\rho=0.27, p=0.51)$. When controlling for the mean cerebral value of subarachnoid space area/skull area ratio, the correlation between the total callosal/skull area ratio and the relative mean cerebral cortical IMZ uptake was still highly significant (partial correlation coefficient $=0.95, \mathrm{p}<0.005)$. The relative mean cerebral cortical IMZ uptake was

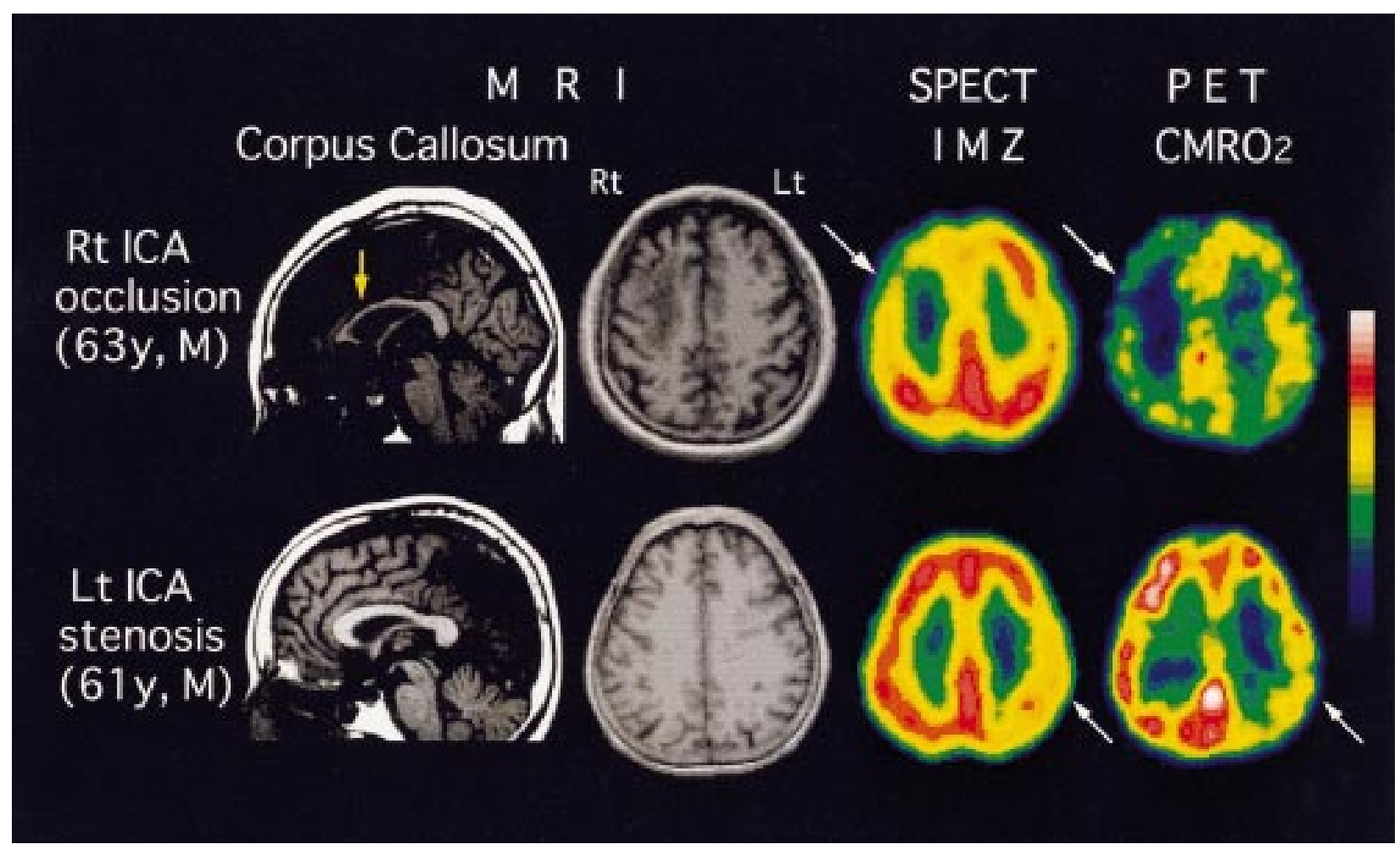

Figure 1 Examples of corpus callosum atrophy as depicted on T1 weighted images (TR=400 ms, TE=15 ms) (first column), and SPECT images, from which was derived the relative iomazenil uptake (IMZ, according to a pseudocolour scale ranging from 0 to $150 \%$ ) (third column), PET images, from which was derived the cerebral metabolic rate of oxygen (CMRO, from 0 to $4 \mathrm{ml} / 100 \mathrm{~g} / \mathrm{min})$ (last column), and the corresponding three dimensional MR images $\left(T R=40 \mathrm{~ms}, T E=5 \mathrm{~ms}\right.$, flip angle $=40^{\circ}, F O V=24 \mathrm{~cm}$ ) (second column). Upper row: severe atrophy of the corpus callosum with anterior predominance (arrow) and decreases in IMZ uptake and $\mathrm{CMRO}_{2}$ accentuated in the right frontal cortex (arrows); Lower row: mild atrophy with mild decreases in IMZ uptake and $\mathrm{CMRO}_{2}$ in the left parietal cortex (arrows). 


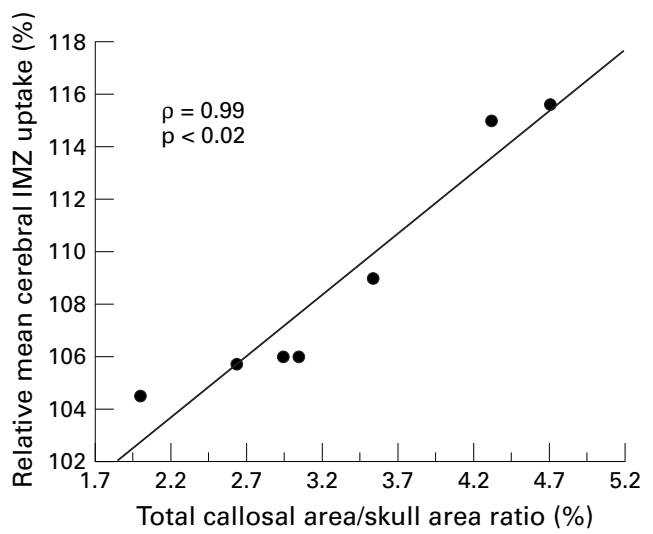

Figure 2 Scatter diagram plotting the total callosal area/skull area ratio against the relative mean cerebral cortical IMZ uptake. The relative IMZ uptake is the ratio of the cerebral IMZ uptake to the mean IMZ uptake in the bilateral cerebellar cortices. The line was generated by simple linear regression.

not correlated with the mean cerebral value of the $\operatorname{CBF}(\rho=0.18, p=0.66)$.

When the hemispheric values of the relative IMZ uptake, the $\mathrm{CMRO}_{2}$, the subarachnoid space area/skull area ratio, the ventricular area/ skull area ratio, the white matter lesion scores, the patient age, and the time between the last symptoms and the MRI evaluation were entered in a stepwise regression analysis, the hemispheric values of the relative IMZ uptake in the bilateral hemispheres accounted for a significant proportion of the variances of the total callosal/skull area ratio, with adjusted $\mathrm{R}^{2}=0.81$; the other variables did not significantly contribute to the magnitudes of this $\mathrm{R}^{2}$.

\section{Discussion}

This study showed that atrophy of the corpus callosum is associated with a decrease in the cerebral cortical BZR binding in large cerebral arterial occlusive diseases with only minor subcortical infarction in the chronic stage. We found that the total callosal area/skull area ratio was significantly decreased in patients compared with control subjects. The severity of callosal atrophy varied, but was strongly correlated with the decrease of the relative mean cerebral cortical IMZ uptake. Stepwise regression analysis showed that, among the decreased relative IMZ uptake, white matter lesions, cortical atrophy, ventricular dilatation, and decreased cortical oxygen metabolism, the decrease in the relative IMZ uptake was the most important independent factor for callosal atrophy.

There are several possible mechanisms for corpus callosum atrophy with decreased cortical BZR binding in large cerebral arterial occlusive diseases. A decrease in callosal size may result from primary ischaemic changes of the corpus callosum, or axonal degeneration from cortical neuronal loss or white matter damage. On the other hand, a decrease in IMZ uptake may result from cortical neuronal loss of ischaemic origin, neuron degeneration due to white matter/callosal infarcts, terminal degeneration, or receptor down regulation due to undercutting or impaired protein synthesis.
The major cause of callosal atrophy did not seem likely to be primary ischaemic damage of the corpus callosum, because T2 weighted MR images showed no high intensity areas in the corpus callosum in all cases. The corpus callosum is relatively resistant to hypoperfusion. ${ }^{30}$ White matter damage also seemed unlikely to be the major cause, because the correlation of cortical IMZ uptake with callosal size was independent of the degree of white matter damage. Previous studies also showed that subcortical infarct dose not necessarily associated with decreased BZR binding in the overlying cerebral cortex. ${ }^{15}{ }^{20}$ Although a functional down regulation of the BZR in the perilesional tissue may be caused by focal cortical lesions, ${ }^{31}$ a simple down regulation does not cause callosal atrophy, if it does not accompany the processes which result in neuronal death. Thus, the best explanation of the parallel decrease in callosal size and IMZ uptake may be primary cortical damage leading to loss of the neurons. ${ }^{2}$ The vulnerability of cortical layer 3 may explain the finding that the degree of callosal atrophy was greater than that of the decreases in IMZ uptake. ${ }^{1}$ The magnitude of the decrease of IMZ uptake may reflect the degree of neuronal damage among all cortical layers, whereas the magnitude of callosal atrophy may reflect the degree of the loss of the neurons in layer 3. However, we cannot exclude completely the possibility of secondary cortical neuronal degeneration or synaptic changes caused by axonal damage in the white matter, because the severity of axonal damage may not correlate with the degree of high intensities on T2 weighted MR images.

In patients with large cerebral arterial occlusive diseases, all the white matter damage, cortical atrophy, decreased cortical oxygen metabolism, and decreased cortical IMZ uptake may be associated with callosal atrophy through cortical neuronal damage, and decreased IMZ uptake may be the best indicator of cortical neuronal damage among these variables. White matter lesions on T2 weighted MRI in the patients studied, may make only a small contribution to callosal atrophy as focal white matter damage. The main constituent of white matter lesions in this study was a confluent high intensity area in the terminal area (deep watershed area). This white matter lesion may indicate the existence of global haemodynamic insufficiency, which may cause diffuse ischaemic changes in the cerebral cortex, leading to callosal atrophy. ${ }^{1132}$ Atrophy of the cerebral cortex may be correlated with cortical neuronal loss, but it may not reflect a mild degree of neuronal loss due to reactive gliosis. Changes in IMZ uptake were detected even in patients with a slight cortical atrophy in this study. Reduced oxygen metabolism in the normal appearing cortical areas may result not only from neuronal damage but also from functional deactivation due to loss of the activating influences from the lesion. ${ }^{33}$ The contribution of the deactivation to the reduced metabolism leads to the weak correlation between cortical oxygen metabolism and callosal size. Decreased IMZ uptake among the 
hypometabolic cortical regions may provide evidence of neuronal damage that could lead to callosal atrophy.

The decrease in cortical IMZ uptake may also have resulted from cortical atrophy through the partial volume effect. However, cortical IMZ uptake was not correlated with the degree of cortical atrophy, and the correlation between callosal size and cortical IMZ uptake was highly significant with adjustment for cortical atrophy. We estimated the degree of cortical atrophy from the three MRI slices corresponding to the three SPECT images analyzed. Thus, to confirm our results, an examination of the effect of atrophy on the measurements by using the methods which involve doing corrections of SPECT data for atrophy based on coregistration of MRI and SPECT and adjusting the difference of resolution between the two studies may be needed. In addition, different structures were used for the measurements of atrophy and of BZR binding in this study. Thus, we cannot exclude the possibility that callosal atrophy and a decrease in IMZ uptake may be two distinct phenomena lacking any causal relation.

In conclusion, atrophy of the corpus callosum is associated with a decrease in cortical BZR binding in large cerebral arterial occlusive disease. Corpus callosum atrophy with decreased cortical BZR binding may reflect cortical neuronal damage, although the entity of neuronal loss without infarction remains to be pathologically confirmed in patients with occlusion of the large cerebral arteries. Callosal atrophy might be a sensitive indicator of ischaemic neuronal loss when considering vulnerability of cortical layer 3, whereas the decrease in IMZ uptake might clearly reflect the regional distribution of neuronal damage. A regional decrease of neurons, which might be shown by decreased IMZ uptake, may be related to focal cortical signs such as aphasia, ${ }^{16}{ }^{17}$ whereas diffuse neuronal loss, which might be shown by callosal atrophy, may be related to global cognitive deterioration. ${ }^{11}$ Thus, the concomitant evaluations of IMZ uptake and callosal size could enhance the power of the assessment of cortical ischaemic damage in patients with large cerebral arterial occlusive diseases.

We thank Dr Yoshiharu Yonekura (Biomedical Imaging Research Center, Fukui Medical School, Fukui, Japan) and the staff of the Department of Radiology and Nuclear Medicine, Faculty of Medicine, Kyoto University for their support and technical help.

1 Graham DI. Hypoxia and vascular disorders. In: Adams JH, Duchen LW, eds. Greenfield's neuropathology. London: Edward Arnord, 1992:153-268.

2 Garcia JH, Lassen NA, Weiller C, et al. Ischemic stroke and incomplete infarction. Stroke 1996;27:761-5.

3 Lassen NA, Losen TS, Højgaard K, et al. Incomplete infarction: a CT-negative irreversible ischemic brain lesion. f Cereb Blood Flow Metab 1983;3 (suppl 1):S602-3.

4 Innocenti GM. General organization of callosal connections in the cerebral cortex. In: Jones EG, Peters A, eds. Cerebral cortex. 5th ed. New York: Plenum Press, 1986:291-353.

5 Powers WJ, Martin WRW, Herscovitch P, et al. ExtracranialPowers WJ, Martin WRW, Herscovitch P, et al. Extracranialintracranial bypass surgery: hemody

6 Gibbs JM, Wise RJS, Leenders KL, et al. Evaluation of cerebral perfusion reserve in patients with carotid artery occlusion. Lancet 1984; i:310-4.

7 Samson Y, Baron JC, Bousser MG, et al. Cerebral hemodynamic and metabolic changes in carotid artery occlusion: a
PET study. In: Meyer JS, Lechner H, Reivich M, Ott EO, eds. Cerebral vascular diseaese. 5th ed. New York: Excerpta Medica, 1985:128-35.

8 Yamauchi H, Fukuyama H, Kimura J, et al. Hemodynamics in internal carotid artery occlusion examined by positron emission tomography. Stroke 1990;21:1400-6.

9 Yamauchi $\mathrm{H}$, Fukuyama $\mathrm{H}$, Nabatame $\mathrm{H}$, et al. Callosal atrophy with reduced cortical oxygen metabolism in carotid artery disease. Stroke 1993;24:88-93.

10 Yamauchi H, Pagani M, Fukuyama $\mathrm{H}$, et al. Progression of atrophy of the corpus callosum with deterioration of cerebral cortical oxygen metabolism after carotid artery occlusion: a follow up study with MRI and PET. $\mathcal{F}$ Neurol Neurosurg Psychiatry 1995;59:420-6.

11 Yamauchi H, Fukuyama H, Nagahama Y, et al. Atrophy of the corpus callosum associated with cognitive impairment and widespread cortical hypometabolism in carotid artery occlusive disease. Arch Neurol 1996;53:1103-9.

12 Abadie P, Baron JC. In vivo studies of the central benzodiazepine receptors in the human brain with positron emission tomography. In: Diksic M, Reba RC, eds. Radiopharmaceuricals and brain pathology studies with PET and SPECT. Boca Ratson, FL: CRC Press, 1991:357-79.

13 Beer HF, Blauenstein PA, Hasler PH, et al. In vitro and in vivo evaluation of iodine-123-Ro 16-0154: a new imaging agent for SPECT investigations of benzodiazepine receptors. F Nucl Med 1990;31:1007-14.

14 Olsen RW. The GABA postsynaptic membrane receptorionophore complex. Site of action of convulsant and ionophore complex. Site of action of convulsant and

15 Sette G, Baron JC, Young AR, et al. In vivo mapping of brain benzodiazepine receptor changes by positron emission tomography after focal ischemia in the anesthetized baboon. Stroke 1993;24:2046-57.

16 Hatazawa J, Satoh T, Shimosegawa E, et al. Evaluation of cerebral infarction with iodine 123-iomazenil SPECT. $f$ Nucl Med 1995;36:2154-61.

17 Hatazawa J, Shimosegawa E, Satoh T, et al. Central benzodiazepine receptor distribution after subcortical hemorrhage evaluated by means of [123I] iomazenil and SPECT. Stroke 1995;26:2267-71.

18 Dong Y, Fukuyama H, Nabatame H, et al. Assessment of benzodiazepine receptors using iodine-123-labeled iomazenil single-photon emission computed tomography in patients with ischemic cerebrovascular disease. A comparison with PET study. Stroke 1997;28:1776-82.

19 Nakagawara J, Sperling B, Lassen NA. Incomplete brain infarction of reperfused cortex may be quantitated with iomazenil. Stroke 1997;28:124-32.

20 Sasaki M, Ichiya Y, Kuwabara Y, et al. Benzodiazepine receptors in chronic cerebrovascular disease: comparison with blood flow and metabolism. F Nucl Med 1997;38: 1693-8.

21 Moriwaki H, Matsumoto M, Hashikawa K, et al. Iodine123-iomazenil and iodine-123-iodoamphetamine SPECT in major cerebral artery occlusive disease. $\mathcal{F} \mathrm{Nucl} \mathrm{Med}$ 1998;39:1348-53.

22 Yamauchi H, Fukuyama H, Nagahama Y, et al. Atrophy of the corpus callosum, cognitive impairment, and cortical hypometabolism in progressive supranuclear palsy. Ann Neurol 1997;41:606-14.

23 Onishi Y, Yonekura Y, Tanaka F, et al. Delayed image of iodine-123 iomazenil as a relative map of benzodiazepine receptor binding: the optimal scan time. Eur 7 Nucl Med 1996;23:1491-7.

24 Sadato N, Yonekura Y, Senda M, et al. PET and the autoradiographic method with continuous inhalation of oxygen5-gas: theoretical analysis and comparison with conventional steady-state methods. F Nucl Med 1993;34:1672-80.

25 Frackowiak RSJ, Lenzi GL, Jones T, et al. Quantitative measurement of regional cerebral blood flow and oxygen metabolism in man using ${ }^{15} \mathrm{O}$ and positron emission tomography: theory, procedure, and normal values. $7 \mathrm{Com}$ put Assist Tomogr 1980;4:727-36.

26 Lammertsma AA, Jones T. Correction for the presence of intravascular oxygen-15 in the steady-state technique for measuring regional oxygen extraction ratio in the brain: 1 . Description of the method. F Cereb Blood Flow Metab 1983; 3:416-24.

27 Friston KJ, Ashburner J, Frith CD, et al. Spatial registration and normalization of images. Human Brain Mapping 1995; 2:165-89.

28 Kretschmann HJ, Weinrich W. Neuroanatomy and cranial computed tomography. New York: Thieme, 1986:70-4.

29 Yamauchi H, Fukuyama H, Kimura J. Hemodynamic and metabolic changes in crossed cerebellar hypoperfusion. Stroke 1992;23:855-60.

30 Moody DM, Bell MA, Challa VR. The corpus callosum, a unique white matter tract: anatomic features that may explain sparing in Binswanger disease and resistence to flow to fluid masses. AfNR Am $\mathcal{F}$ Neuroradiol 1988;9:1051-

31 Witte OW, Stoll G. Delayed and remote effects of focal cortical infarctions: secondary damage and reactive plasticity. Adv Neurol 1997;73:207-27.

32 Yamauchi H, Fukuyama H, Yamaguchi S, et al. Highintensity area in the deep white matter indicating hemodynamic compromise in internal carotid artery occlusive disorders. Arch Neurol 1991;48:1067-71.

33 Feeney DM, Baron JC. Diaschisis. Stroke 1986;17:817-30. 